\title{
Lissencephaly-pachygyria spectrum in a North Indian boy with Wolcott-Rallison syndrome due to homozygous deletion of exon 1 in the EIF2AK3 gene
}

Objawy ze spektrum lissencefalii-pachygyrii u chłopca z Północnych Indii z zespołem Wolcotta-Rallisona z powodu homozygotycznej delecji eksonu $1 \mathrm{w}$ genie EIF2AK3

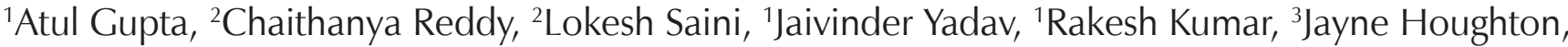 \\ ${ }^{4}$ Sian Ellard, ${ }^{1}$ Devi Dayal \\ ${ }^{1}$ Endocrinology and Diabetes Unit, Department of Pediatrics, Postgraduate Institute of Medical Education \\ and Research, India \\ ${ }^{2}$ Neurology Unit, Department of Pediatrics, Postgraduate Institute of Medical Education and Research, \\ Chandigarh, India \\ ${ }^{3}$ Clinical Genetics, Royal Devon and Exeter NHS Foundation Trust, Exeter, United Kingdom \\ ${ }^{4}$ Institute of Biomedical and Clinical Science, College of Medicine and Health, University of Exeter, Exeter, \\ United Kingdom
}

\begin{abstract}
Background: Wolcott-Rallison syndrome (WRS) is a rare autosomal recessive disorder characterized by neonatal diabetes mellitus (NDM), epiphyseal dysplasia, and hepatic and renal dysfunction. Although neuro-psychological features are common in patients with WRS, malformations of cortical development (MCDs) are rarely reported.

Case presentation: A 3-month-old boy, born to non-consanguineous parents, presented with right focal seizures since two months of age and recently detected diabetes mellitus. He also had a small head and lissencephaly-pachygyria spectrum on brain imaging. Genetic testing confirmed the diagnosis of WRS by identifying a biallelic homozygous deletion of exon 1 in the EIF2AK3 gene. The child achieved reasonable glycemic control on the basal-bolus insulin regimen.

Conclusion: Presentation of WRS may occur with neurological manifestations such as lissencephaly-pachygyria spectrum. Early confirmation of the genetic diagnosis of WRS by screening for pathogenic variants in the EIF2AK3 gene is important in children with NDM and associated syndromic features. Establishing the diagnosis of WRS helps in predicting the development of subsequent clinical features, guides management, and may improve patient outcomes.
\end{abstract}

Key words:

Wolcott-Rallison syndrome, EIF2AK3 gene, neonatal diabetes mellitus, monogenic diabetes, malformations of cortical development.

\section{Introduction}

Wolcott-Rallison syndrome (WRS) (OMIM 226980) is a rare autosomal recessive disease characterized by early-onset diabetes mellitus, epiphyseal dysplasia, hepatic dysfunction, and growth retardation, and results from pathogenic variants in the Eukaryotic Translation Initiation Factor 2-Alpha Kinase 3 (EIF2AK3) gene [1]. Other clinical manifestations include hypothyroidism, exocrine pancreatic insufficiency, renal insufficiency, intellectual deficits, neutropenia, and recurrent infections [2]. Neuro-psychological development is commonly affected, although its severity is variable [1]. The neurological manifesta- tions range from almost normal development to severe neuromotor deficits, intractable epilepsy, intellectual deterioration, and microcephaly [1]. There are rare reports of malformations of cortical development (MCD), such as simplified gyral pattern (SGP), in children with WRS [2]. However, the lissencephalypachygyria pattern has not been reported as part of the clinical phenotype of WRS.

Most cases of WRS present with non-autoimmune and insulin-dependent diabetes during the first six months of age. In populations with high consanguinity rates, WRS is the most common cause of genetic neonatal diabetes mellitus (NDM) [3]. India being a multi-ethnic country, the rates of consanguinity 
vary widely across different regions within the country. Consequently, the occurrence of WRS varies in different geographical areas. In Southern India, WRS is considered the most common cause of NDM, while its occurrence in Northern India is rare $[4,5]$. In this communication, we describe an infant from Northwest India diagnosed as WRS with additional lissencephalypachygyria spectrum features.

\section{Case presentation}

This 3-month-old boy was the first-born baby to nonconsanguineous parents and weighed $2.6 \mathrm{~kg}$ at birth. At two months of age, he developed right focal seizures with varying frequency of 2-15 episodes per day, for which he was treated elsewhere with phenytoin. He was subsequently referred to our hospital after initial investigations showed hyperglycemia (random blood glucose $527 \mathrm{mg} / \mathrm{dl}$ ) and lissencephaly. There was no previous history of a similar disorder in the family. The determination of lack of consanguinity between parents was made on the basis of a pedigree history.

On examination, he weighed $5.2 \mathrm{~kg}(-1.04$ SDS, standard WHO growth chart) and had a small head (head circumference
$38.5 \mathrm{~cm},-2.18$ SDS, standard WHO growth chart). Neurological examination revealed mild limb hypotonia and normal tendon reflexes. The rest of his systemic examination was unremarkable.

Investigations revealed hemoglobin of $10.6 \mathrm{~g} / \mathrm{dl}$, total leukocyte count $13.9 \times 10^{9} /$ I (neutrophils $20 \%$, lymphocytes $72 \%$ ) and platelet count $358 \times 10^{9} /$. The routine biochemistry, including liver and kidney function tests, were within the normal range. Glycosylated hemoglobin $\left(\mathrm{HbA}_{1 \mathrm{c}}\right)$ was $13.1 \%$, and fasting and stimulated C-peptide concentrations were $0.105 \mathrm{ng} / \mathrm{ml}$ (normal range, $0.78-1.89 \mathrm{ng} / \mathrm{ml}$ ) and $0.644 \mathrm{ng} / \mathrm{ml}$ (normal range, $5-12 \mathrm{ng} / \mathrm{ml}$ ), respectively. Plasma adrenocorticotropin hormone and AM cortisol concentrations were $65.35 \mathrm{pg} / \mathrm{ml}$ (normal range, 7.2-63 pg/ml) and $292.9 \mathrm{nmol} / \mathrm{l}$ (normal > $150 \mathrm{nmol} /$ ), respectively. Serum triiodothyronine (T3), thyroxine (T4) and thyroid-stimulating hormone $(\mathrm{TSH})$ concentrations were $0.89 \mathrm{ng} / \mathrm{ml}$ (normal range $0.2-2.0 \mathrm{ng} / \mathrm{ml}$ ), $7.11 \mu \mathrm{g} / \mathrm{dl}$ (normal range, 4.8-12.7 $\mu \mathrm{g} / \mathrm{dl}$ ) and $3.84 \mu \mathrm{lU} / \mathrm{ml}$ (normal range, $0.27-4.2 \mu \mathrm{lU} / \mathrm{ml}$ ) respectively. Pancreatic autoantibody profile showed positive islet antigen 2 (IA2) but negative anti-insulin (IAA) and glutamic acid decarboxylase 65 (GAD 65) antibodies. Electroencephalogram (EEG) showed bilateral posterior pseudo-periodic bursts of intermittent epilepti-
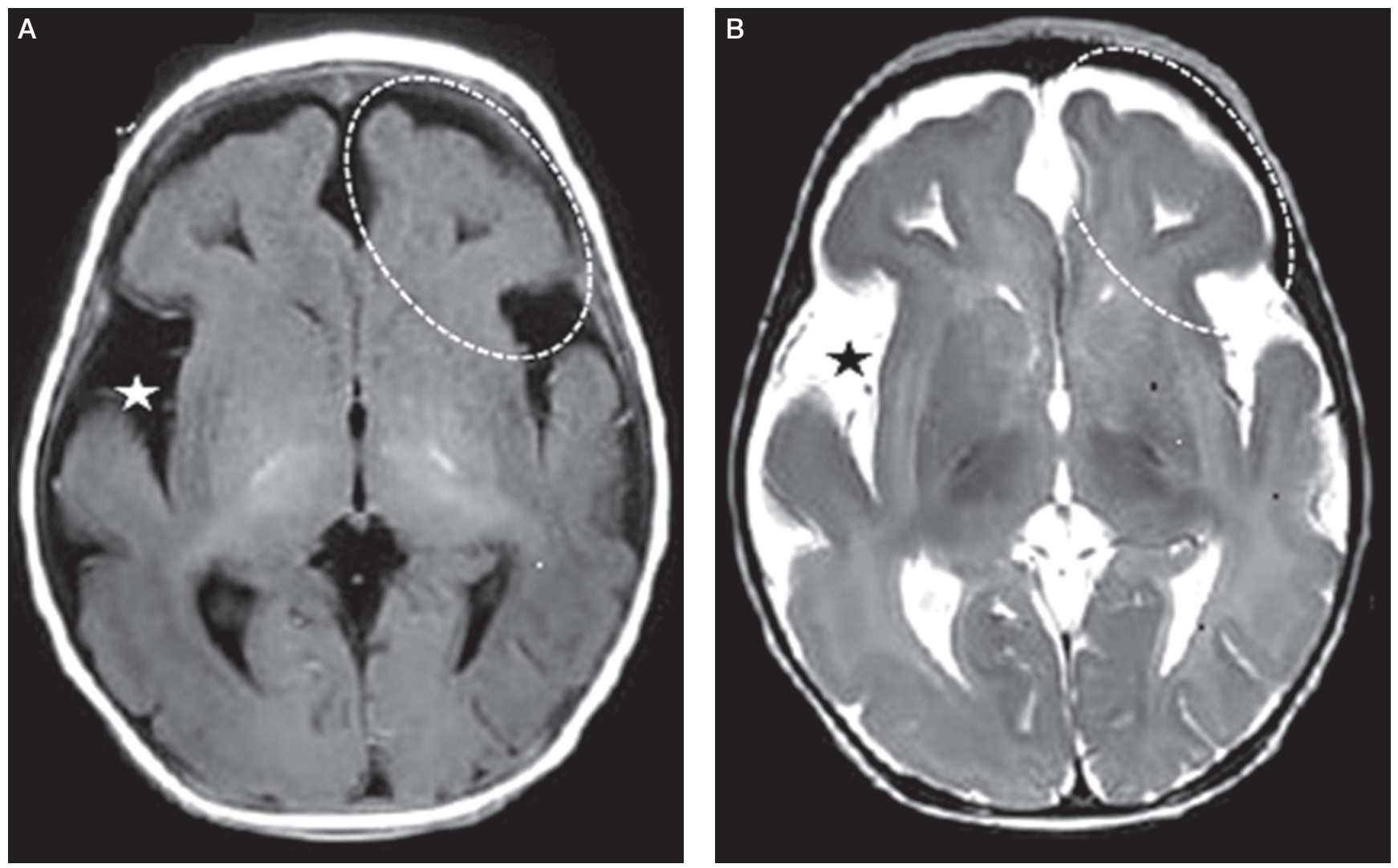

Figure 1. Axial T1-weighted (A) and T2-weighted (B) magnetic resonance images showing anterior predominant thick cortex with simplified gyriform pattern suggestive of lissencephaly-pachygyria complex (dashes oval markers). Mild cerebral atrophy is also evident by widened Sylvian fissures (star markers) 
form discharges with anterior background suppression suggestive of nonspecific diffuse cortical dysfunction and underlying cortical irritative foci in left temporo-occipital region. Magnetic resonance imaging (MRI) of the brain showed anterior predominant thick cortex with SGP suggestive of lissencephaly-pachygyria complex, associated with mild cerebral atrophy and widened Sylvian fissures (Fig. 1 A, B). A monogenic NDM was considered as a provisional diagnosis.

Genomic DNA was extracted from leukocytes in the peripheral blood of the affected child and his parents. Analysis of the coding regions and conserved splice sites of 32 NDMrelated genes was performed by targeted next-generation sequencing (NGS) (Agilent custom capture v5.3/Illumina NextSeq500), which can also detect partial or whole gene deletions and duplications. The NGS revealed a homozygous deletion of exon 1 in the EIF2AK3 gene at location (GRCh37, hg19) chr2:(? 88926479) (88926838 ?) with HGVS description of NM 004836.5:c.(? 46) (308+6 309 50) del, p.?. Biallelic lossof-function variants in the EIF2AK3 gene are a known cause of WRS [6]. Confirmation testing was done with dosage analysis of exons 1 and 2 of the EIF2AK3 gene by Droplet Digital PCR using EvaGreen. Carrier testing revealed a heterozygous state for the pathogenic EIF2AK3 deletion variant in both parents. Appropriate informed consent was obtained from the parents for the laboratory studies and genetic tests. The departmental review board approved the study for publication.

The baby was initiated on a basal-bolus insulin regimen. His total insulin dose is 0.5 units/kg/day. At his latest evaluation at six months of age, he weighed $7.0 \mathrm{~kg}(-1.23 \mathrm{SDS})$, and his $\mathrm{HbA}_{1 \mathrm{c}}$ was $8.8 \%$.

\section{Discussion}

Children with WRS often present with NDM, which is an essential criterion for diagnosis [1]. Other clinical features such as skeletal dysplasia, growth retardation, hepatic, kidney, and thyroid dysfunction, neutropenia, and neurological manifestations appear as the age advances [1]. We expect that a periodic screening by radiological imaging, biochemical, hormonal, and hematological tests will reveal additional features in our patient well in time to offer appropriate management. The highlight of our case is the presence of unusual neurological manifestations in the form of the lissencephaly-pachygyria spectrum on brain imaging. Neuro-psychological manifestations are the second most common symptoms in WRS seen in almost two-thirds of patients [7]. Yet, the MCD, such as gyral pattern abnormalities, are noted rarely. One of the two previous cases, who showed MCD, was reported to have SGP on neuroimaging, while the other had pachygyria and cerebral atrophy at 21 months of age $[2,8]$. Our patient is probably the youngest to show a pattern of lissencephaly-pachygyria. Interestingly, he has not shown signs of developmental regression yet. It has been observed previously that developmental regression may occur as late as 30 months of age and is particularly common after hepatic failure episodes begin to appear [7, 8]. The mechanism of neurological abnormalities is the same as the other organ dysfunctions in WRS. In normal circumstances, upon endoplasmic reticulum (ER) stress, EIF2AK3 detects the accumulation of misfolded proteins in the ER, phosphorylates EIF2 $\alpha$, and downregulates the rate of protein synthesis [1]. EIF2 $\alpha$ phosphorylation results in the expression of the $\mathrm{CHOP}$, a transcription factor that performs multiple functions during ER stress. The homozygous loss-of-function deletion variant in the EIF2AK3 gene makes it non-functional, resulting in the accumulation of a large amount of misfolded proteins in affected cells and tissues that leads to increased apoptosis. The increased apoptosis causes premature cell death, responsible for the organ dysfunction in WRS $[1,7]$.

There are some unusual features of diabetes in our patient. Typical diabetes in WRS is non-autoimmune [1, 3, 4]. The IA2 autoantibody positivity at three months of age in our patient indicates pancreatic autoimmunity, even though the main pancreatic autoantibodies commonly observed in our patient population of autoimmune diabetes were not detected $[9,10]$. The frequency of pancreatic autoantibodies in the general pediatric population is extremely low, and even a single autoantibody positivity is linked with the future development of autoimmune diabetes [11]. Other characteristic features of diabetes in WRS, such as onset with DKA, poor glycemic control, and frequent occurrence of severe hypoglycemia, were not observed in our patient over the short observation period [4, 12]. The glycemic control may, however, show worsening over the long-term, similar to the experience in large patient cohorts [12].

Although WRS is a common cause of NDM in consanguineous families, its occurrence is extremely rare in NDM children born to non-consanguineous parents. None of the 11 children with genetically confirmed NDM previously reported from Northwest India had WRS [5]. There is, however, a recent report of a novel disease-causing variant of the EIF2AK3 gene in another child from our region [13]. At our center, the index patient is the second case of WRS out of 34 patients with NDM registered over the last 15 years, 12 of whom have genetically confirmed monogenic diabetes $[14,15]$. This is in sharp contrast to reports from Southern India, where WRS is a common diagnosis in children with NDM, probably due to higher consanguinity rates in their population $[4,6]$.

In conclusion, we report a biallelic homozygous deletion of exon 1 in the EIF2AK3 gene in an infant with features of WRS that included NDM and an unusual neurological manifestation of lissencephaly-pachygyria spectrum. Screening for pathogenic genetic variants in the EIF2AK3 gene in all patients with NDM allows early diagnosis of WRS, which is vital for providing genetic counseling, optimizing management, and predicting the development of additional manifestations with this syndrome. 


\section{References}

1. Julier C, Nicolino M. Wolcott-Rallison syndrome. Orphanet J Rare Dis 2010; 5: 29. doi: 10.1186/1750-1172-5-29.

2. de Wit MC, de Coo IF, Julier C, et al. Microcephaly and simplified gyral pattern of the brain associated with early onset insulin-dependent diabetes mellitus. Neurogenetics 2006; 7: 259-263. doi: 10.1007/s10048-006-0061-1.

3. Abbasi F, Habibi M, Enayati S, et al. A Genotype-First Approach for Clinical and Genetic Evaluation of Wolcott-Rallison Syndrome in a Large Cohort of Iranian Children With Neonatal Diabetes. Can J Diabetes 2018; 42: 272-275. doi: 10.1016/j.jcjd.2017.06.009.

4. Varadarajan P, Sangaralingam T, Senniappan S, et al. Clinical profile and outcome of infantile onset diabetes mellitus in southern India. Indian Pediatr 2013; 50: 759-763. doi: 10.1007/s13312-0130219-5.

5. Jain V, Satapathy A, Yadav J, et al. Clinical and Molecular Characterization of Children with Neonatal Diabetes Mellitus at a Tertiary Care Center in Northern India. Indian Pediatr 2017; 54: 467-471. doi: 10.1007/s13312-017-1049-7.

6. Rubio-Cabezas O, Patch AM, Minton JA, et al. Wolcott-Rallison syndrome is the most common genetic cause of permanent neonatal diabetes in consanguineous families. J Clin Endocrinol Metab 2009; 94: 4162-4170. doi: 10.1210/jc.2009-1137.

7. Ozbek MN, Senée V, Aydemir S, et al. Wolcott-Rallison syndrome due to the same mutation (W522X) in EIF2AK3 in two unrelated families and review of the literature. Pediatr Diabetes 2010; 11 : 279-285. doi: 10.1111/j.1399-5448.2009.00591.x.

8. Iyer S, Korada M, Rainbow L, et al. Wolcott-Rallison syndrome: a clinical and genetic study of three children, novel mutation in EIF2AK3 and a review of the literature. Acta Paediatr 2004; 93: 1195-1201.
9. Dayal D, Samprati M, Kaur N, et al. Prevalence of Beta-Cell, Thyroid and Celiac Autoimmunity in North Indian Children with Recent Onset Type 1 Diabetes (T1D). J Clin Diagn Res 2015; 9: SM01-2. doi: 10.7860/JCDR/2015/11960.5711.

10. Dayal D, Hegde A. Prevalence of Islet Autoantibodies in Type 1 Diabetes. Indian J Endocrinol Metab 2017; 21: 485-486. doi: 10.4103/ ijem.IJEM_24_17.

11. Batstra MR, Petersen JS, Bruining GJ, et al. Low prevalence of GAD and IA2 antibodies in schoolchildren from a village in the southwestern section of the Netherlands. Hum Immunol 2001; 62: 1106-1110. doi: 10.1016/s0198-8859(01)00283-x.

12. Welters A, Meissner T, Konrad K, et al. Diabetes management in Wolcott-Rallison syndrome: analysis from the German/Austrian DPV database. Orphanet J Rare Dis 2020; 15: 100. doi: 10.1186/ s13023-020-01359-y.

13. Bhaskar V, Thunga C, Tiwari S, Ellard S, Flanagan S. Wolcott-Rallison Syndrome due to a Novel Mutation in EIF2AK3 Gene. 55th Annual Meeting of the European Society for Paediatric Endocrinology (ESPE), Paris, France, September 10-12, 2016: Abstracts. Horm Res Paediatr 2016;86 Suppl 1: 1-556.

14. Dayal D, Saini AG, Jayashree M, et al. Hospital based incidence, patterns of presentation and outcome of Type 1 diabetes: 12 years' data from a tertiary care center in North India. Int J Diabetes Dev Ctries 2015; 35: 103-107.

15. Kumar R, Pilania RK, Verma D, et al. Infantile onset diabetes mellitus: fifteen years' experience from a tertiary care pediatric center in North India. Annual Meeting of Sri Lanka College of Endocrinologists, Colombo, Sri Lanka, August 1-3, 2019: Abstracts. Sri Lanka J Diab Endocrinol Metab 2019; 9 suppl 1. Available at: https://www. researchgate.net/publication/341399444. 